\title{
On the noise and physical realizability of experimental polarimetric images
}

\author{
Y. Takakura, J. El Sayed Ahmed, M.-Ph. Stoll and J. Zallat \\ LSIIT, Université de Strasbourg, 67412, Illkirch, France
}

\begin{abstract}
Optical pol arimetric im ages are us ually c alculated from a set of int ensity images re corded with different states of polarization of light [1]. Be cause th ese latter s tates are setup dependen t, p ropagation of experimental noise to polarimetric images is governed by the architecture of the polarimeter. In the case of Mueller polar imeter with ro tating wave $\mathrm{p}$ lates, polarimetric no ise is dis tributed ac cording to a scheme that involves 4 unco rrelated blocks of $2 \times 2$ adjacen t matrices, each matrix containin g 4 correlated elements [2] . When minimal $4 \times 4=16$ intensity images are used to extract Mueller images, the 4 elements in the middle m22, $\mathrm{m} 23, \mathrm{~m} 32, \mathrm{~m} 33$ fea ture $\mathrm{v}$ ariances that are 4 times higher th an the $\mathrm{v}$ ariance of the intensity measurements. These highes $t$ polarimetric variances only equalize the in tensity variance when $8 \times 8=64$ intensity images are used for as sessment. Polarimetric a ccuracy r eferred to as "physical realizability" of M ueller images can be checked on the spectrum of a particular 4x4 real matrix built with the Minkowsk i space tensor G= $\operatorname{diag}\left(\left[\begin{array}{lll}1 & 1 & 1\end{array}\right.\right.$ 1]) together with the Mueller matrix $M$ [3]. Because the defined estimator has undesirable statistical properties such as an unfa vorable bias, any test on $\mathrm{M}$ ueller images of non depolarizing samples would lead to negativ e results almost all the time. A correct checking procedure would be to take into account the previously exhibited properties of po larimetric no ise. This is formally equivalent for overpolarizing tests, to consid er a correctly computed shifted ellipsoid rather than the usual centered sphere [4].
\end{abstract}

\section{References}

1. J. L. Pezzaniti and R. A. Chipman, Opt. Eng., 34, 1558 (1995)

2. Y. Takakura and J. El Sayed Ahmad, Appl. Opt., 46, 7354 (2007)

3. C. R. Givens and A. B. Kostinski, J. Mod. Opt., 40, 471 (1993)

4. Y. Takakura and M.-Ph. Stoll, Appl. Opt., 481073 (2009) 